\title{
Short Note on Unification of Field Equations and Probability
}

\author{
Mesut KAVAK
}

\begin{abstract}
Is math in harmony with existence? Is it possible to calculate any property of existence over math? Is exact proof of something possible without pre-acceptance of some physical properties? This work is realized to analysis these arguments somehow as simple as possible over short cuts, and it came up with some compatible results finally. It seems that both free space and moving bodies in this space are dependent on the same rule as there is no alternative, and the rule is determined by mathematics.
\end{abstract}

\section{Introduction}

It seems that matter is uncertain. Uncertainty brings some physical properties out together with itself. Matter emerges as a density over time by increasing acceleration as waves over free space being has time differences between any point of its free space. Space turns into particles and particles turn into space continual manner. Matter gains its mass by collecting space in a limited volume at light speed. During its emergence it constantly experiences potential difference since there are time differences in the other name emergence priority. There is a single work to create all the universe, and the work is done one by one for each point of free space and thus particles. By this way any point of the universe gets the same speed since the single work is done by the same speed as work-done is equal to kinetic energy. Total energy of matter is according to this creation work. Because of this reason matter cannot go faster than light as also cannot move at lower speeds since the light speed is the highest speed as also the lower is not possible. When an object moves from A to B in space, it means inner motion or in the other name its formation motion which brings energy for matter out during its continuous creation, moved to A to B at the creation speed. Matter uses the same space at the same time [1].

While matter experiences the potential differences, naturally there emerge some denser and low density and thus disordered or more ordered points. Disordered points want to be distributed on lower density or more ordered space points since have more stress. Matter gains its total energy because of the work done against this resistance of free space, that otherwise it would not be created since the existent smallest force can move the existent bigger mass magnitude at infinite speed.

The sliding space from disorder to more ordered point also takes the space points or particles together with itself since the they emerge over this sliding free space. Actual reason of force and energy is this gravity is included, and force is only distributed as waves over time along space.

\section{Impossibility of perpendicularity}

There is a representation of an Euclidean geometry. In this case, being $\mathrm{BD}$ and $\mathrm{BC}$ are fixed, assume that $\mathrm{AB}$ is lengthened to any other $\mathrm{AB}_{2}$ length. Here $\mathrm{A}$ point can be assumed as moving body in free space, $\mathrm{B}$ point is a fixed point which its coordinate is known in space and $\mathrm{C}$ is an observer.

For the lengthened hypotenuse on Fig. 1, over the inequality and definition of $\mathrm{AB}_{2}>\mathrm{AB}$, it becomes (1) over the inequality of $\mathrm{BD}_{2}^{2}-\mathrm{BD}^{2}>\mathrm{AD}^{2}-\mathrm{AD}_{2}^{2}$,

$$
1>\frac{\mathrm{AD}^{2}-\mathrm{AD}_{2}^{2}}{\mathrm{BD}_{2}^{2}-\mathrm{BD}^{2}}
$$

where $\mathrm{BD}^{2}+\mathrm{AD}^{2}=\mathrm{AB}^{2}$ and $\mathrm{BD}_{2}^{2}+\mathrm{AD}_{2}^{2}=\mathrm{AB}_{2}^{2}$ are the equations over Pythagorean theorem. In the same manner, it

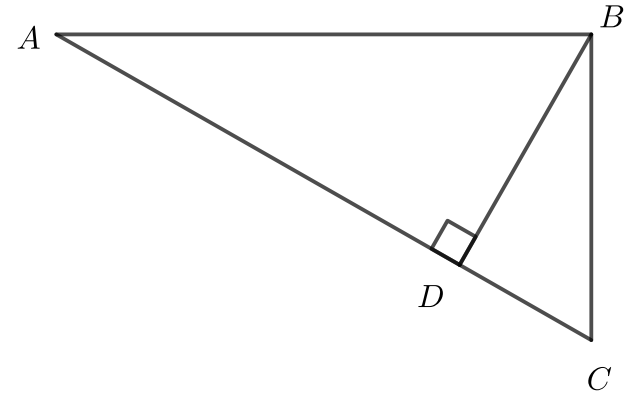

Fig. 1: This is an Euclidean right triangle. Euclidean geometry is the geometry which areas, angles and lengths are related as equality together with whole and certain numbers and there are no complex, uncertain or irrational numbers, irrational numbers are with a limit. Instead of this right triangle, it can be used a triangle which there is no right angle occurs in it. The shape is random. It does not mean actual geometry. For example, BD length can be longer than AD length in the below stated calculations.

becomes (2),

$$
\mathrm{BD}^{2}+\mathrm{DC}^{2}=\mathrm{BD}_{2}^{2}+\mathrm{DC}_{2}^{2}
$$

where $\mathrm{h}^{2}+\mathrm{t}^{2}=\mathrm{y}^{2}$ and $\mathrm{h}_{2}^{2}+\mathrm{t}_{2}^{2}=\mathrm{y}^{2}$ are the equations over Pythagorean theorem. If (2) is edited, it becomes $\mathrm{DC}^{2}$ $\mathrm{DC}_{2}^{2}=\mathrm{BD}_{2}^{2}-\mathrm{BD}^{2}$; thus if $\mathrm{DC}^{2}-\mathrm{DC}_{2}^{2}$ is used instead of $\mathrm{BD}_{2}^{2}-\mathrm{BD}^{2}$ on (1), also it becomes (3) over $\mathrm{DC}^{2}-\mathrm{DC}_{2}^{2}>$ $\mathrm{AD}^{2}-\mathrm{AD}_{2}^{2}$ inequality.

$$
1>\frac{\mathrm{AD}^{2}-\mathrm{AD}_{2}^{2}}{\mathrm{DC}^{2}-\mathrm{DC}_{2}^{2}}
$$

Now the actual displacement inequalities have been determined. Right this point, assume that there is no displacement, namely there is no lengthening. For this condition, it becomes $\mathrm{AB}_{2}=0, \mathrm{DC}_{2}=0$ and $\mathrm{AD}_{2}=0$; thus (3) becomes (4),

$$
\mathrm{DC}^{2}>\mathrm{AD}^{2}
$$


and (1) becomes (5).

$$
-\mathrm{BD}^{2}>\mathrm{AD}^{2}
$$

The inequality of (5) actually means it is impossible to be $\mathrm{AD}=\mathrm{DC}$ that means perpendicularity is impossible in universe. This also means that at the same time no lengths can be the same. From physical perspective, it means each point of free space has the same speed and energy magnitude at the end of 1 second but the same time, and emergence is one by one for the total of universe. Each point emerges by order. This also means, for any force-applied, since there is no middle point the natural motion is always circular as there is no alternative. Because of circular displacement, centrifugal force is always together with motion.

The inequality of (5) has another results, and are as the below stated ones.

- Exactly there is no middle point place for any size part of area; so a motion is always circular for any forceapplied.

- A right angle cannot emerge. It is only close to right angle due to the energy which area holds.

- It cannot be drawn two line segments which have the same length from a point in space to other two points. Namely, 3 or more objects cannot take place in space being the distance between each of them is the same. There is a time difference between each point of space at the same assumed global time.

- For $\mathrm{AD} \neq \mathrm{DC}$, it also becomes $\mathrm{AB} \neq \mathrm{BC}, \mathrm{BD} \neq \mathrm{DC}$ $\mathrm{BD} \neq \mathrm{AD}, \mathrm{BD} \neq \mathrm{AB}$ and more. Namely, while $\mathrm{AB}$ is lengthened, $\mathrm{BC}$ or the other lengths cannot protect own actual length. The medium is conservative. This also means, that length and thus 1 dimension do not exist alone; because 2 objects cannot take place at a distance relatively to each other. Namely, length is not absolute since is relative. Length occurs in a limited time interval and gets lost constantly, and cannot be independent on speed. Higher dimension parts of lower dimensions have different size than lower dimensional parts. Namely a 2 dimensional square cannot be used by the same size to create a 3 dimensional cube.

- The shortest distance between 2 objects is not a line segment. This distance is an arc so close to a line segment.

- Parallel two line segment cannot be drawn beyond drawing line segment. They are exactly intersected, and the intersection point occurs according to area multitude of conservative area. By this way, no object can protect its speed. There is always an opposite space force to decelerate. By this way, as the same is acceptable for the existent smallest infinite part as well, constant speed motion or constant acceleration motion is not possible. Motion emerges by parts, and is always with small acceleration parts has frequency changing over time, and the result is average as assumed constant speed, and is seen as constant speed.

- A closed curve is not possible. Only infinite space closes curve. Limited space is not closed but is conservative.

- Motion cannot be independent of medium whatever the distance between moving bodies. This means, that there is an absolute entanglement between area and lengths in area although there is time differences. It shows one-piece behavior. This is only possible with emergence one by one. Namely, all mass is a single mass with emergence priority. Each point occurs by order. In this way, information is conserved forever; because flexible collision is not possible. There never occurs a mutual extinction which is because of decrease in the energy and mass by certain, whole numbers. Instead of it, during collisions, energy and mass values get smaller to infinite small part as irrational numbers; but it never disappears.

\section{Uncertainty}

If area is conservative then for 2 dimensional medium, it becomes Eq. (6),

$$
\mathrm{dxdy}=0
$$

where $\mathrm{ds}=\mathrm{dx}+\mathrm{dy}, \mathrm{ds}^{2}=\mathrm{dx}^{2}+\mathrm{dy}^{2}$ and thus $\mathrm{ds}=\mathrm{ds}^{2}$. Here, even if the two components always change, sum of them is always conserved for any physical value for example as $\mathrm{p}=$ $\mathrm{p}_{\mathrm{x}}+\mathrm{p}_{\mathrm{y}}$ and $\mathrm{p}^{2}=\mathrm{p}_{\mathrm{x}}^{2}+\mathrm{p}_{\mathrm{y}}^{2}$. It means $2 \mathrm{D}$ motion is not possible.

In $3 \mathrm{D}$ space, the equation becomes Eq. (7),

$$
\mathrm{dx}\left(\frac{1}{\mathrm{dy}}+\frac{1}{\mathrm{dz}}\right)=-\frac{1}{2}
$$

where $\mathrm{ds}^{2}=\mathrm{dx}^{2}+\mathrm{dy}^{2}+\mathrm{dz}^{2}$ and $\mathrm{ds}=\mathrm{dx}+\mathrm{dy}+\mathrm{dz}$.

There are five possibilities for motion's emergence type constantly or partially by some intervals as $d x=d y=d z$, $\mathrm{dx}=\mathrm{dy}, \mathrm{dx}=\mathrm{dz}$ and $\mathrm{dy}=\mathrm{dz}$ or none of them. $\mathrm{dx}=\mathrm{dy}=$ $\mathrm{dz}$ equation is not possible when it is checked over Eq. (7). For the others, if dx is taken from Eq. (7), and then if it is put on its place on equation which is $\mathrm{ds}=\mathrm{dx}+\mathrm{dy}+\mathrm{dz}$, the equation becomes Eq. (7a).

$$
\mathrm{ds}=\mathrm{dy}+\mathrm{dz}-\frac{\mathrm{dy} \cdot \mathrm{dz}}{2(\mathrm{dy}+\mathrm{dz})}
$$

Here, assume that it is $\mathrm{ds}^{2}=\mathrm{dx}^{2}+\mathrm{dy}^{2}$ which is the projection of the same ds during forming a sphere by $\mathrm{ds}^{2}=$ $\mathrm{dy}^{2}+\mathrm{dz}^{2}$, where $\mathrm{dx}=\mathrm{dz}$ by the same angle vertically and horizontally according to a fixed reference; then Eq. (7a) becomes Eq. (7b).

$$
d x^{2}=d y \cdot d z+d z^{2}-\frac{d y^{2} \cdot d z^{2}}{4(d y+d z)^{2}}
$$

Over Eq. (7b), it can be said that $d x=d y$ and $d x=d z$ are not possible. For $\mathrm{dy}=\mathrm{dz}$ equality, it becomes $\mathrm{dx} / \mathrm{dy}=33 / 16$; but if it is used on the main function Eq. (7), it seems that even it is not possible as well; therefore there is only one possibility left that none of them emerges even for any interval of motion, and the components are always different. They never intersect for any combination. Any point of free space has emergence priority due to the time differences

Over the defined elements, presentation turns into Fig. 2.

Fig. 2 is actually an attractor. It can only be valid in this shape at the beginning of time since the distance taken was all possible ways of the universe in the smallest time. As you can see over the above stated derivative components, $\mathrm{dx}$, $\mathrm{dy}, \mathrm{dz}$ are the smallest 3D derivative components of an unknown function whatever the function is; so as matter basically works over the same principle, namely if that components were for speed, distance, force or time by changing functions, the rule would be the same even component magnitudes change; so according to these component magnitude change, the attractor naturally changes for selected different intervals; but at the end, any motion closes to the rule of attractor.

Warning 


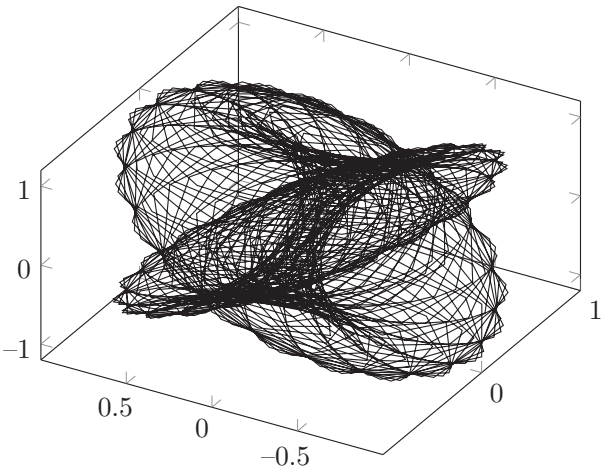

Fig. 2: Limited presentation of mass emergence by the parametric function which I derived [1].

\section{Existence probability of uncertain bodies}

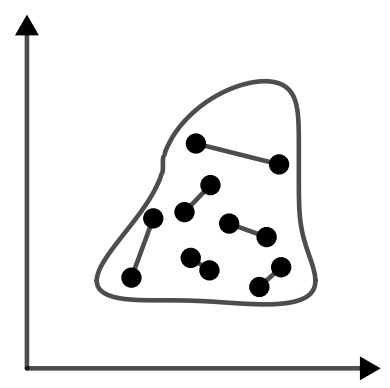

Fig. 3: Conservative area with a random closed curve

There is a random shaped closed curve on Fig. 3. Even if $2 \mathrm{D}$ is not possible, we are going to work on 3D over it.

Now think that some changing magnitude forces are applied to create mass in this area between two point. Any small point of are has emergence priority; thus they can never intersect even for increasing points; so if we turn the Fig. 3 into Fig. 4, it is also acceptable.

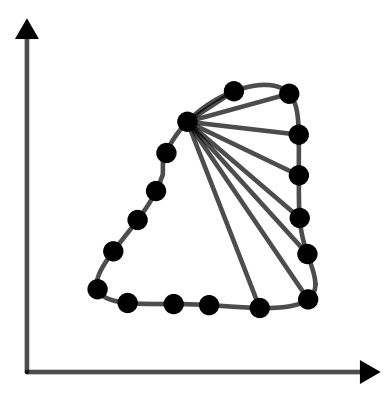

Fig. 4: Creating mass

On Fig. 4, assume that the force is applied from a point to the other all point on the circumference being the distance between the points are the same. For this, as there is emergence priority and thus time difference for each small point of area; being $\mathrm{n}$ is total point number on the circumference, it can be $\mathrm{n} \Delta \mathrm{l}_{1}$ as also can be $\mathrm{n}^{2} \Delta \mathrm{l}_{2}$ where $\Delta \mathrm{l}$ is average length of the used to create an area; thus it becomes $\Delta \mathrm{l}_{1}=\mathrm{n} \Delta \mathrm{l}_{2}$ over $\mathrm{n}^{2} \Delta \mathrm{l}_{2}=\mathrm{n} \Delta \mathrm{l}_{1}$. It means, if you draw all lines only from one point to the other all point, you need more points on the circumference to scan or create and thus to converge the area. If you draw lines from each point to the other all points, then you do not need more points on the circumference, and both of them are acceptable since there is not going to be intersection point of lines as stated the above. In the same manner, the equation also becomes Eq. (8),

$$
\mathrm{nE}=\mathrm{A}
$$

where $\mathrm{E}=$ Fr over average length on $\lim _{\mathrm{r}_{0} \rightarrow \mathrm{r}}\left(\mathrm{F}_{1} \mathrm{r}_{0}+\mathrm{F}_{2} \mathrm{r}_{0}+\right.$ $\left.\ldots+\mathrm{F}_{\mathrm{n}} \mathrm{r}_{0}\right)$ is total energy and $\mathrm{A}$ is total area. Here $\mathrm{E}$ and A does not converge over $\lim _{n \rightarrow \infty}$; instead of it $\mathrm{A}$ is always fixed; so if you write $\lim _{\mathrm{n} \rightarrow \infty} \mathrm{E}=\mathrm{A}$, it means zero energy which the area $\mathrm{A}$ on the horizon holds. To understand this, assume that you have a square has $r$ side in accordance with abstract math. If you draw the closest parallel $\mathrm{r}$ lines to this side, you obtain the square and its $\mathrm{r}^{2}$ area; but also if you draw the lines as perpendicular, you obtain $2 \mathrm{r}^{2}$ area. If you constantly repeat it, being $\mathrm{f}$ is repeat number, it has $\mathrm{fr}^{2}$ area; but in accordance with the time difference, it cannot exist such since there cannot be intersection point; so since there are infinite time differences and thus space allows this, during the process, the energy which the area holds decreases as it can be seen over Eq. (8) being the area is the same. This creates a density concept such as energy density or mass density which the area holds. Area does not specify energy, distance or mass. Assume that there are infinite kind of free space in absolute space; then area is the part which you closed on horizon as imaginary to calculate the physical values of the space which is in the borderlines of the area you closed. Space could be created in any way by changing physical values as independent on the area you closed. As a result, $\mathrm{E}$ is dependent on $\mathrm{n}$ inversely proportional being $\mathrm{E}$ is $\mathrm{E}(\mathrm{n})$.

Any curve which is assumed as closed can also be defined in the kind of a circle for $\mathrm{r}=\sqrt{\mathrm{A} / \pi}$ radius over $\mathrm{A}=\pi \mathrm{r}^{2}$. Already any closed curve must be circle at the end if there is motion. Namely, if you take center of gravity for closed curves as a reference, it becomes $\left|\mathrm{F}_{\mathrm{p}_{+} \mathrm{x}} \mathrm{r}_{+\mathrm{x}}-\mathrm{F}_{\mathrm{p}_{-} \mathrm{x}} \mathrm{r}_{-\mathrm{x}}\right|>0$ in absolute value where $r_{+x} \neq r_{-x}$ and $F_{p}$ is partial force applied on a line in the area; but it becomes $\left|\mathrm{F}_{\mathrm{p}_{+} \mathrm{x}} \mathrm{r}_{+\mathrm{x}}-\mathrm{F}_{\mathrm{p}_{+} \mathrm{x}}\right| \approx$ 0 in absolute value where $r_{+x}=r_{-x}$ since the path for any force-applied is the same in circle as radius, and $\mathrm{F}_{\mathrm{p}}$ is partial force applied on a line in the area. It is approximate 0 since there is actually time differences and thus actually for the same time also force magnitude or distance taken cannot be the same in different direction. As a result, it becomes $\left|\mathrm{F}_{\mathrm{p}_{+} \mathrm{x}} \mathrm{r}_{+\mathrm{x}}-\mathrm{F}_{\mathrm{p}_{-} \mathrm{x}} \mathrm{r}_{-\mathrm{x}}\right|>\left|\mathrm{F}_{\mathrm{p}_{+} \mathrm{x}} \mathrm{r}_{+\mathrm{x}}-\mathrm{F}_{\mathrm{p}_{+} \mathrm{x}}\right|$ in absolute value. The difference in force in closed curves which are not circle also creates motion in accordance with the definition of force since it will be $\left(\mathrm{F}_{1}-\mathrm{F}_{2}\right) / \mathrm{t}=\mathrm{v}_{\mathrm{F}}$ where $\mathrm{t}$ is time of change in force and $v_{F}$ is its velocity or will be $m_{1} a_{1}-m_{2} a_{2}=m a$; so they are stressed and they want to take themselves to lower energy state, and at the end, to minimum energy state for its any point since denser points has more energy; but as it was said, this creates motion.

Hence the other variation of $\Delta \mathrm{l}_{1}=\mathrm{n} \mathrm{l}_{2}$ and thus of Eq. (8) as $\mathrm{nA}=\mathrm{E}$ is impossible; because matter moves from the denser space to lower density naturally. Forcing it to denser space is also moving to lower density since still matter emerges at that time by moving to lower density in inner space. Namely, natural characteristic of matter is not dependent on $\mathrm{nA}=\mathrm{E}$ since for the fixed $\mathrm{A}$, it is going to be $\lim _{\mathrm{n} \rightarrow \infty} \mathrm{E}=\infty$. It cannot allow emergence of motion. 


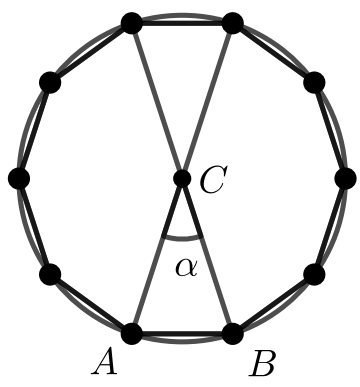

Fig. 5: Polygon transformation being $n$ is even number

Over Fig. 5, it becomes Eq. (9) where $\alpha=360 / \mathrm{n}$ and $\mathrm{AB}=\mathrm{d}$ and $\mathrm{E}_{2}=\mathrm{nFr} / \mathrm{n}$ assuming $\mathrm{n}$ goes to infinite.

$$
\mathrm{d}^{2}=2 \mathrm{E}_{2}^{2}-2 \mathrm{E}_{2}^{2} \cos (\alpha)
$$

Also it becomes Eq. (10) over nd $=2 \pi \sqrt{\mathrm{A} / \pi}$ for the circumference of the circle where $\pi \mathrm{r}^{2}=\mathrm{A}$ assuming $\mathrm{n}$ goes to infinite.

$$
\mathrm{d}^{2}=\frac{4 \pi \mathrm{A}}{\mathrm{n}^{2}}
$$

If Eq. (9) and Eq. (10) are equalized to each other, then we get Eq. (11),

$$
1-\cos (\alpha)=\frac{2}{\mathrm{r}^{2}}
$$

where $\alpha=360 / \mathrm{n}$. Here assume that $\cos (\alpha)$ is the sum of all possibilities of possible existence of $\mathrm{F}$ and $\mathrm{r}$ relatively to each other since $\cos (\alpha)$ changes over $\alpha=360 \mathrm{~F} / \pi \mathrm{r}$ where $\mathrm{n}=\mathrm{A} / \mathrm{E}$. Namely all possible $\mathrm{F}$ and $\mathrm{r}$ values are in this area for $\int \cos (\alpha)$ dr. Also you can use $\int(1-\cos (\alpha)) \mathrm{dr}$ as an option. For $\int \cos (\alpha)$ dr, we have Eq. (12),

$$
1=\frac{360 \mathrm{x}}{\pi} \operatorname{Si}\left(\frac{360 \mathrm{x}}{\pi \mathrm{y}}\right)+\mathrm{y} \cos \left(\frac{360 \mathrm{x}}{\pi \mathrm{y}}\right)
$$

where $\mathrm{x}=\mathrm{F}$ and $\mathrm{y}=\mathrm{r}$. Do not think the functions allow $\mathrm{n}=\infty$; because it creates infinite probabilities. In the exact opposite way, matter is uncertain as convergent. Namely $n$ has an end. Otherwise matter would not be created since E is going to become 0 ; so for the calculation $\pi$ is going to be a decimal number for required approximation.

\subsection{A special condition for time}

As it was said, matter is uncertain; but as area is conservative in accordance with the proven impossibility of perpendicularity over the right triangle at the beginning of the paper, derivative resultant always becomes $\mathrm{ds}=1$ for any high dimension number due to $\mathrm{ds}^{\mathrm{n}}=\mathrm{ds}$; so for example it is always $\mathrm{mc}=1$ as a certain number; but as emerging matter has time differences it becomes Eq. (13),

$$
\frac{1}{1-\mathrm{t}_{0}}>1
$$

where $t_{0}$ is relative time of any selected space point according to any other point being $1 \mathrm{sec}$. is total time of the entire universe or as relatively is the time of another point when the others have not get it. It creates an uncertainty. The creator of the universe can only know the certainty. The inner elements can- not know energy or time being certain. Each physical property that time is included as well, exactly converges to a value being having different length decimals after the comma for any point of the universe continuously. By this way information is conserved forever since there cannot be a flexible collision or friction by the same natural numbers, integers or for example the irrational numbers with a limit as real numbers. Hence even if total physical values of the universe is certain, it is assumed as uncertain by these rules. Otherwise infinite frequency in a limited area renders impossible to emerge of matter being created.

Actually this is a progressive explanation of the Copenhagen Interpretation; because as long as there is an interaction element that you can call it as observer, matter emerges. At the times when a relation appears, matter appears together with the relation. Observer does not have to be living thing. Any point of free space has emergence priority and thus has different speeds at the same time since constant speed is not possible as it was proven [1]. There is only one work to create all the universe, and any point gets the same speed of the work-done to create universe for 1 second of each space point, not at the same time. Each point is counted as observer. The light speed is not fixed for any reference system $[1,3]$ at the same moment. Also it can slows down that actually this must be the actual expectation of change in time. Namely speed does not slow; but is a result of distribution on area. The creation work is over area, and the work is scanning. Speed must be understood in this manner. Namely, if observational speed slows, it means there is more area in the medium to scan, that there is denser space part. At this condition, you close to the smallest creation time.

As will power, it has a second effect on matter; because natural flow of motion is dependent on some natural physical rules; but you can change the natural flow by will.

As a result, matter has no absolute threshold value. Any small force can move any big mass instantly. At the moments when a measurement is done or an interaction emerged, matter is certain as it converges to a number. It appears from imaginary time that all infinite information is there without motion [1], does work and gets smaller forever as irrational value by converging and under the estimated threshold value for a limit. Infinite frequency condition is this. Otherwise matter could not appear with infinite frequency. This is the explanation of the motion which does not repeat itself even in a limited interval. By this way, information get smaller forever but its loss, and constantly converges to a distance value forever. You think it stooped but not. Like the sea waves, it comes ashore as waves even in free space under the estimated threshold value as required by conservation of information. There is no flexible collision because of the time differences. At the same moment, any point has different mass, time and velocity.

\subsection{A special condition for probability}

Actually we can write Eq. (11) as Eq. (14) in radiant,

$$
1=\alpha+\cos (\alpha)
$$

where $\alpha=2 / \mathrm{r}^{2}$ over $\mathrm{n}=\mathrm{A} / \mathrm{E}$ and $\mathrm{F}=1 / \mathrm{r}$. We can write it as $\mathrm{F}=1 / \mathrm{r}$; because actually over $\mathrm{nFr}=\mathrm{A}$ equality, $\mathrm{A}$ is always fixed and thus we can use it as $\mathrm{A}=1$. n can only changes the magnitude; so the basic rule between Fandr is $\mathrm{F}=1 / \mathrm{r}$. Maybe we can use this in this manner.

Also force or distance is not continuous on $\mathrm{x}=\mathrm{F}$ and $\mathrm{y}=\mathrm{r}$ or $\mathrm{x}=\mathrm{r}$ and $\mathrm{y}=\mathrm{F}$ axis for any calculation method. Even if you take the values like $\mathrm{x}=\mathrm{F} / \mathrm{r}$ and $\mathrm{y}=\mathrm{r}$ or $\mathrm{x}=$ $\mathrm{r}$ and $\mathrm{y}=\mathrm{F} / \mathrm{r}$, the rule must be the same. When one of them become 0 , the other one can exist but requires another 
dimension. It is not a paradox or calculation mistake since there is a $3 \mathrm{D}$ motion.

The wave equation of Schrödinger seems a general representation. Namely it seems like an attractor. It does not give the actual shape of atom. Even, if you accept electron as wave, it is very dangerous that math may interpret the creation frequency as electron is there and here being there is absolute emptiness between the places since matter is not continuous. Namely, you know that number pi has all credit numbers and passwords; but if you find the one which belongs to someone, you must investigate the conditions which cause the number. The same may be valid for field equations. Namely you can make an inference like black holes over the geometric rules of math by field equations; but do not forget it is like an intersection point of any probability. It is like a break point. These seem only for motivation instruments about what is going on there to draw a road map.

\section{Warning}

Already $\mathrm{F}=0$ is estimated result since we cannot know both of them at the same moment together, as it was proven above over derivative components.

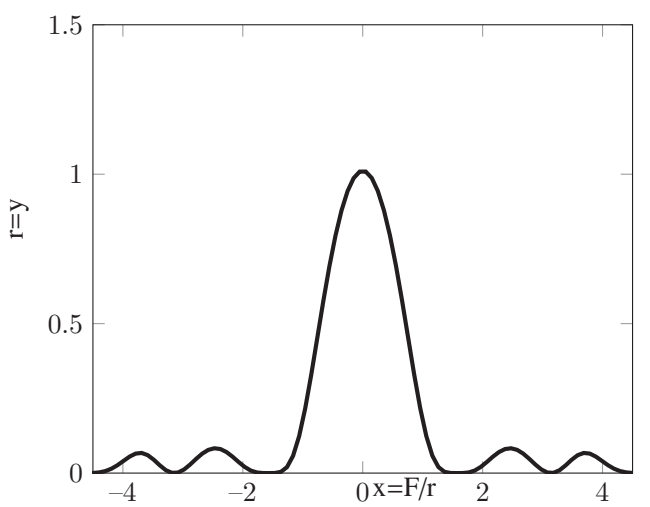

Fig. 6: Representation of change in existence of force and distance.

For the second option, over $\int 1-\cos (\alpha) \mathrm{dr}=\int \frac{2 \mathrm{~F}}{\mathrm{r}^{2}} \mathrm{dr}$, it becomes Eq. (15),

$$
\mathrm{y}=\frac{\frac{180 \mathrm{xy}}{\pi} \operatorname{Si}\left(\frac{360 \mathrm{x}}{\pi \mathrm{y}}\right)}{1-\cos \left(\frac{360 \mathrm{xy}}{\pi \mathrm{y}}\right)}
$$

where $x=F / r$ and $r=y$. Since is $y=f(x, y)$, do not make a simplification for $\mathrm{y}$ and accept it as $\mathrm{f}(\mathrm{x}, \mathrm{y})=1$; so it turns into Eq. (16).

$$
\mathrm{y}=\frac{\pi\left(-1+\cos \left(\frac{360 \mathrm{x}}{\pi}\right)\right.}{180 \mathrm{x} \operatorname{Si}\left(\frac{360 \mathrm{x}}{\pi}\right)}
$$

For this equation, both force and distance become zero now and again it means, there is a circular motion and dimensional change. Already because of the time differences, there cannot be a middle point as also was proven at the beginning; so for any force-applied, a circular motion emerges.

\section{Area effect on optics}

As total energy is dependent of $\mathrm{nE}=\mathrm{nFr}=\mathrm{mc}^{2}$, it becomes Eq. (17).

$$
\lim _{\mathrm{n} \rightarrow \infty} \frac{\mathrm{mc^{2 }}}{\mathrm{nr}}=\mathrm{F}=0
$$

Since force is sum of both circular displacement and moving to more ordered area at the same time together because of time differences, it actually means $\mathrm{F}_{\mathrm{G}}+\mathrm{F}_{\mathrm{C}}=0$ and thus means Eq. (18),

$$
\mathrm{F}_{\mathrm{G}}=-\mathrm{F}_{\mathrm{C}}
$$

or meansEq. (19) over $\lim _{\mathrm{n} \rightarrow \infty} \frac{\mathrm{mc}^{2}}{\mathrm{nr}}=\mathrm{F}_{\mathrm{C}}+\mathrm{F}_{\mathrm{G}}$ where $\mathrm{F}_{\mathrm{C}}=$ $\mathrm{mc}^{2} / \mathrm{r}$.

$$
\lim _{n \rightarrow \infty} \frac{m c^{2}}{r}\left(\frac{1}{n}-1\right)=-\frac{m c^{2}}{r}=F_{G}
$$

It means, matter can move in two different type as distributed on area or as focused since when $\mathrm{n}$ is infinite, it is like a linear motion; but actually $n$ cannot be infinite, for focusing, you can only see the part of a higher dimension that actually is $3 \mathrm{D}$ in lower dimension that actually is 2D due to the changing values of in an interval that it does not have to be for only creating, that is possible after creation for focal points of universe over partial forces or distances of total values. These also mean that the actual equation is Eq. (20),

$$
\mathrm{F}_{\mathrm{G}} \approx-\mathrm{F}_{\mathrm{C}}
$$

where $\mathrm{F}_{\mathrm{G}}>\mathrm{F}_{\mathrm{C}}$ or $\mathrm{F}_{\mathrm{C}}>\mathrm{F}_{\mathrm{G}}$ that one of them may always valid, or by changing intervals there is a constant transformation from one of them to the other one due to conditions.

Here actually 2D means both $1 \mathrm{D}$ and 3D. Actually it means still 3D; but depth and width are too close to a threshold value; so also you can assume that is 1D instead of 2D since it is going to be counted as without area or volume. Also it seems that it is related with the famous mass gap of Yang-Mills.

\section{Warning}

How we can describe the equality or the inequality as axial? It can only become Fig. 7, and it is insufficient even if the angles can change anywise.

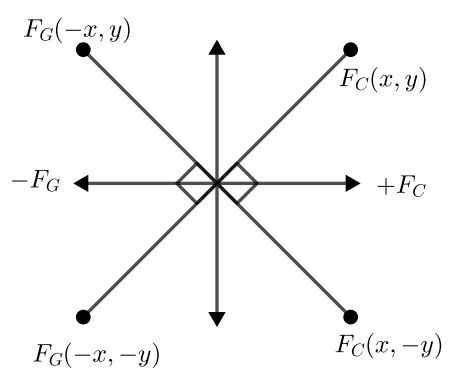

Fig. 7: Gravity and centrifugal force

It is insufficient; because on one axis, only one of them can be placed. It means Riemann surfaces are in the question, and complex analysis is required. The symmetry in the universe seems central symmetry.

Namely if I should show both focusing and complex placement together, it becomes Fig. 8. Here real and imag- 


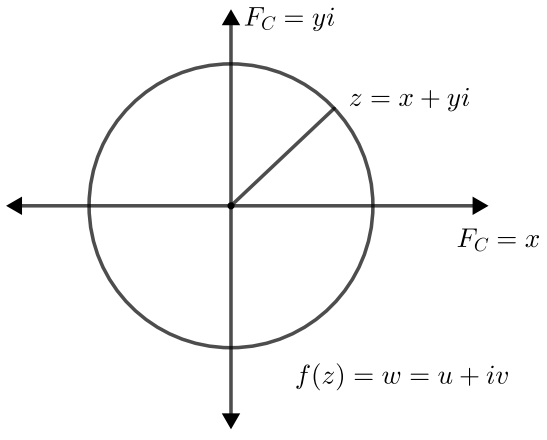

Fig. 8: Complex plane

inary parts are intersected at the middle of the quarter circle. Somehow, it is like it, that neither imaginary part nor real part does not increase from 0 or does not decrease to 0 . Just the point slides slowly since is $\mathrm{F}_{\mathrm{G}} \approx-\mathrm{F}_{\mathrm{C}}$. Here is like an intersection point of motion between dimensions. Finally over it, it becomes Fig. 9.

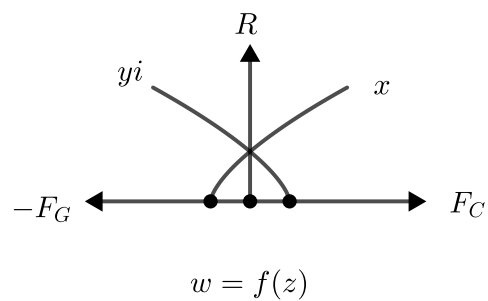

Fig. 9: Complex plane for the image set

As the focusing again, for example over Fig. 10, the focused matter appears more around black and white area instead of the other grey colors between black and white, according to your duplicating direction. This shape is only for example. It is not identical.

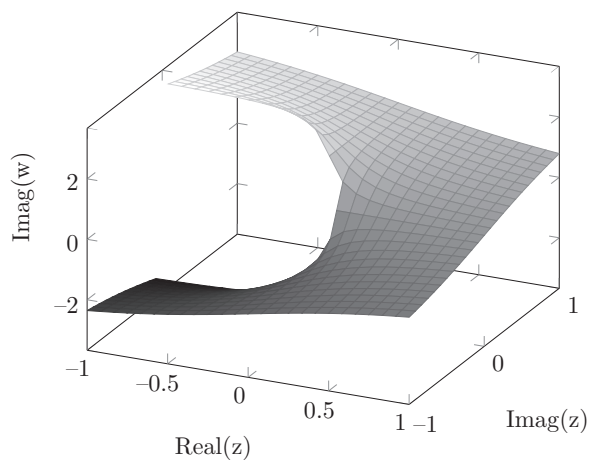

Fig. 10: Single branch of a random Riemann surface

\section{Area effect on gravitational behaviour: The local grav- itational fields}

There is an object which is assumed as doing circular or elliptic motion around a mass on Fig. 11.

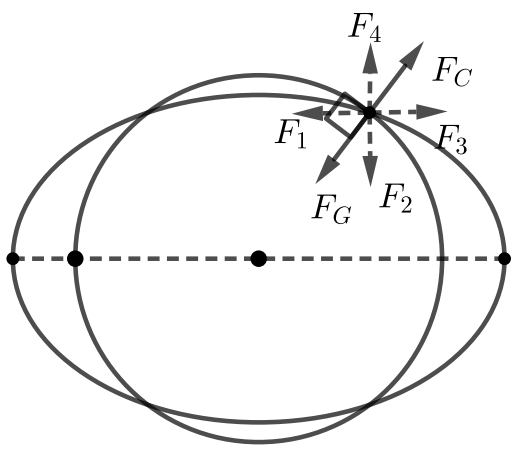

Fig. 11: Circular and elliptic orbits

For this condition, it becomes $\mathrm{F}_{\mathrm{G}}=\sqrt{\mathrm{F}_{1}^{2}+\mathrm{F}_{2}^{2}}$ and $\mathrm{F}_{\mathrm{C}}=\sqrt{\mathrm{F}_{3}^{2}+\mathrm{F}_{4}^{2}}$. Over $\mathrm{F}_{\mathrm{G}}=\mathrm{F}_{\mathrm{C}}$ assumption, it becomes Eq. (21).

$$
\mathrm{F}_{1}^{2}+\mathrm{F}_{2}^{2}=\mathrm{F}_{3}^{2}+\mathrm{F}_{4}^{2}
$$

It means, that for the worst possibility, the components cannot be equal to each other to emerge of motion. If any two of them become equal to each other, then all of them becomes the same force; thus it renders impossible emergence of motion since they are not in the same direction. As a result, we can easily say that it is either $\mathrm{F}_{\mathrm{C}}=\mathrm{F}_{\mathrm{G}}$ with different components or $\mathrm{F}_{\mathrm{C}} \approx \mathrm{F}_{\mathrm{G}}$ again with different components. This conditions render impossible to move on a circular or elliptic orbit; because it must spin being the distance between the object changes over time because of the resultant force even for fixed magnitude different components or for the components which get different values by changing over time, that these two option seems they change escape or falling time and direction; but not. They cannot fly off or fall; because for each attraction or centrifugal force change over $\mathrm{F}_{\mathrm{C}}=\mathrm{F}_{\mathrm{G}}$ equality is conserved; so if escaping force is caused by centrifugal force, at the same magnitude, an attraction emerges. It means, the components change, and an orbit which closes a curve emerges. It cannot perfect circle because of acceleration motion since before a new force is applied with different magnitude and way, the previous force causes acceleration and distance taken.

Orbital objects constantly experience different distances while spinning around a mass. There is no constant boosting. There is a constant transformation between attraction and centrifugal force.

\section{Inference}

Motion cannot be constant speed motion. Objects either accelerate or decelerate constantly or by some changing periods, by using both of them at different times with different magnitudes.

In accordance with conservation of energy, it must be $\mathrm{F}_{1}+$ $\mathrm{F}_{2}+\mathrm{F}_{3}+\mathrm{F}_{4}=\mathrm{F}_{\mathrm{G}}+\mathrm{F}_{\mathrm{C}}$; thus over $\left(\mathrm{F}_{1}+\mathrm{F}_{2}+\mathrm{F}_{3}+\mathrm{F}_{4}\right)^{2}=$ 
$\left(\mathrm{F}_{\mathrm{G}}+\mathrm{F}_{\mathrm{C}}\right)^{2}$, centrifugal force becomes Eq. (22),

$$
\mathrm{F}_{\mathrm{C}}=\frac{\mathrm{F}_{1}\left(\mathrm{~F}_{2}+\mathrm{F}_{3}+\mathrm{F}_{4}\right)+\mathrm{F}_{2}\left(\mathrm{~F}_{3}+\mathrm{F}_{4}\right)+\mathrm{F}_{3} \mathrm{~F}_{4}}{\sqrt{\mathrm{F}_{1}^{2}+\mathrm{F}_{2}^{2}}}
$$

where $\mathrm{F}_{\mathrm{G}}=\sqrt{\mathrm{F}_{1}^{2}+\mathrm{F}_{2}^{2}}$, and gravitational force becomes Eq. (23),

$$
\mathrm{F}_{\mathrm{G}}=\frac{\mathrm{F}_{1}\left(\mathrm{~F}_{2}+\mathrm{F}_{3}+\mathrm{F}_{4}\right)+\mathrm{F}_{2}\left(\mathrm{~F}_{3}+\mathrm{F}_{4}\right)+\mathrm{F}_{3} \mathrm{~F}_{4}}{\sqrt{\mathrm{F}_{3}^{2}+\mathrm{F}_{4}^{2}}}
$$

where $\mathrm{F}_{\mathrm{C}}=\sqrt{\mathrm{F}_{3}^{2}+\mathrm{F}_{4}^{2}}$. It again seems fixed orbital is not possible.

As it can be seen, here $\mathrm{F}_{1}\left(\mathrm{~F}_{2}+\mathrm{F}_{3}+\mathrm{F}_{4}\right)+\mathrm{F}_{2}\left(\mathrm{~F}_{3}+\mathrm{F}_{4}\right)+$ $\mathrm{F}_{3} \mathrm{~F}_{4}$ must be fixed even for higher dimensions for the same rule, since $\mathrm{F}_{\mathrm{G}}=\mathrm{F}_{\mathrm{C}}$ is the equality and thus denominators change the resultant. Over these, we can write $\mathrm{F}_{\mathrm{G}} \mathrm{F}_{\mathrm{C}}=1$ and $\mathrm{F}_{\mathrm{G}}+\mathrm{F}_{\mathrm{C}}=0$; so gravity becomes Eq. (24),

$$
\mathrm{F}_{\mathrm{G}}=\frac{\mathrm{F}_{\mathrm{C}}+1}{\mathrm{~F}_{\mathrm{C}}-1}
$$

and centrifugal force becomes Eq. (25).

$$
\mathrm{F}_{\mathrm{C}}=\frac{\mathrm{F}_{\mathrm{G}}+1}{\mathrm{~F}_{\mathrm{G}}-1}
$$

For the resultant force it becomes Eq. (26).

$$
\mathrm{R}=\sqrt{\left(\mathrm{F}_{4}-\mathrm{F}_{2}\right)^{2}+\left(\mathrm{F}_{3}-\mathrm{F}_{1}\right)^{2}}
$$

In accordance with Eq. (26), natural motion is circular motion that the difference is too small with regular circle; because $\mathrm{F}_{1}$ and $\mathrm{F}_{2}$ can get very small number near zero; so the orbital acceleration which is because of the components, that even if the component magnitudes do not change over time, the resultant over Eq. (26) causes acceleration over $\mathrm{F}=\mathrm{ma}$, causes continuous speed increase in the same orbit without flying off or falling until the existence of the orbital mass creates a density around the central object since already is a resultant of attraction and centrifugal force. When it gets the maximum speed, it means either it became light speed, or since mass emerges for 1 second, its existence is distributed on space and thus speed could not increase to light speed but a different speed limit as attraction properties changed for an unit of time over mass. Hence if an orbital is not circular, it means exactly there is an external drive.

Additionally, it can be seen over real solutions of Eq. (26) that are $F_{1}=F_{3}-R$ and $F_{2}=F_{4}$ or $\mathrm{F}_{1}=\mathrm{F}_{3}+\mathrm{R}$ and $\mathrm{F}_{2}=\mathrm{F}_{4}$. Over these, exactly $F_{1}$ and $F_{3}$ cannot be equal to each other but $\mathrm{F}_{2}=\mathrm{F}_{4}$ is equal; so $\mathrm{F}_{1}^{2}+\mathrm{F}_{2}^{2}=\mathrm{F}_{3}^{2}+\mathrm{F}_{4}^{2}$ equation renders impossible to emerge of motion since becomes 0 over $\mathrm{F}_{2}=\mathrm{F}_{4}$. It means equality is not possible and fixed orbits are not possible since it requires $\mathrm{F}_{\mathrm{C}} \approx \mathrm{F}_{\mathrm{G}}$ or $\mathrm{R} \approx$ $\sqrt{\left(\mathrm{F}_{4}-\mathrm{F}_{2}\right)^{2}+\left(\mathrm{F}_{3}-\mathrm{F}_{1}\right)^{2}}$. It means that also perfect circle cannot be drawn by orbital objects. It is only close to regular circle. It is always elliptic.

\section{Warning}

Over Fig. 11, as it can be seen, for example while $\mathrm{F}_{3}$ is increasing $\mathrm{F}_{1}$ cannot increase or if $\mathrm{F}_{4}$ is increasing $\mathrm{F}_{3}$ cannot increase in accordance with Eq. (26) and $\mathrm{F}_{\mathrm{C}}=\mathrm{F}_{\mathrm{G}}$ equality.
Resultant force which determines the shape of orbit cannot be fixed since there is an acceleration motion and thus there are changing magnitude components over time. It means, after some distance is taken in the way of centrifugal force, again distance is taken in the opposite way, in the direction of gravity. As the change in component magnitude is continuous, it draws sinusoidal wave on the orbit as a second effect. If is $3 \mathrm{D}$, it draws helix around the central mass.

Every orbital object follows helical path which has circular or elliptic orbit at its center.

\section{Inference}

\section{Natural relativistic mass}

In accordance with these findings, gravity is almost equal to centrifugal force if you do not include the time differences that as it was proven [2], if you include it, as long as distance increased, centrifugal force increases, and is the reason of dark matter misunderstanding. There is no dark matter. Linear attraction or momentum turns into angular momentum. This is not boosting; but is transformation. Orbital objects cannot fly off; but they get faster. Gravity does not work as Newtonian. Distance and gravitational force relation changes over distance. The attraction properties change for each point of free space, and have some limits. The attraction changes due to some values between $1 / \mathrm{r}$ and $1 / \mathrm{r}^{2}$

Since mass is gained by motion and motion is circular, centrifugal force as a result of circular displacement causes some special attraction properties in mass creation.

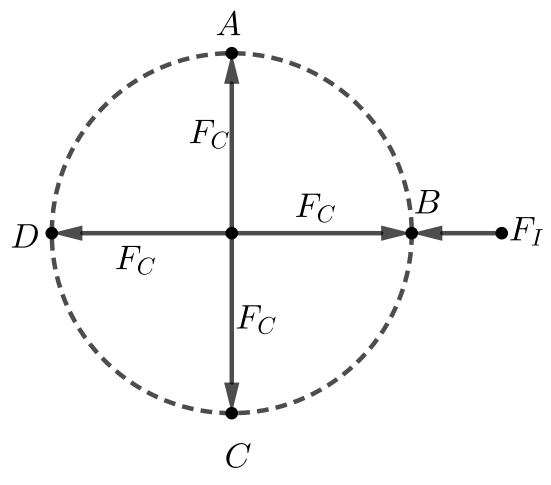

Fig. 12: Natural relativistic mass

Mass is a varying phenomenon, and as a result of this, also attraction is varying. It can seen some times like an external energy is working. Namely you can suppose that energy is not conservative since incredibly small energies can do work much more than their total energies. This is possible, and is dependent on some conditions.

Imagine that the 2D circle is a particle on Fig. 12. Naturally a single wave with a fixed mass and energy winds it, and the particle gains more mass if the frequency gets higher since detected mass of the basic wave in an unit of volume increases. Know that shape can be different, and actually it cannot be static since force is not continuous at every point of the circumference at the same time. It draws circular paths on space; but assume it is static. It is not important now.

Now if you apply a force to push it from B point, if the particle has enough frequency on the surface, naturally no object can pass from $B$ point since any point on the circumference will be like it is full of mass even is not; but if the 
frequency is low relatively, exactly required particles can pass inside of the particle without collision or friction with the surface wave. When $\mathrm{F}=\mathrm{ma}$ is handled, the mass here is the result of the condition. Namely some directions of the centrifugal force of the surface wave are in the same direction with the external force-applied in outer space over $\mathrm{F}=\mathrm{ma}$; but also some of them are in the opposite direction. Hence when you talk about mass, it can only sensed and detected in this way; then think that this particle is spinning around another object like in atoms. What happens if the direction of external force over $\mathrm{F}=$ ma becomes the same with the other object's direction of surface wave. For this condition, relative mass decreases. They get the smallest work value since inertial resistance gets closer to zero for both attraction and repulsion, that means almost zero mass. This depends on how fast they spin around each other, and how many mass they have. Atomic and sub-atomic particles may be inclined to spin in a state of resonance; thus also the emerging attraction or repulsion incredibly increases. This increase is such an increase that no oil can do this. Also resonance is not required. It can occur between some limits by changing values.

It seems that electrons can also lower the mass of nucleus by this way if it gets enough spinning speed and thus frequency, as also can increase. It also means that if you induce an electric charge on a material by AC current not DC, then if you catch the correct frequency, you can make matter worked by its own energy by a phenomenon like almost teleportation against gravity. You do not need so much energy. Even incomparably small energy can realize this. Timing and frequency change everything. It also means, earthquakes can be created without ELF waves or atmosphere can be used to send electricity over air molecules almost without resistance to the other side of the world by small energies. Even it can be focused.

\section{Conclusion}

These show us again, that the phenomena are also chaotic, emerge in an interval but in many different kind. Chaos is a perfect principle, and is a law according to me and my findings.

Also it seems entropy is an absolute law. Any measurable physical value of matter is uncertain. Even for after the comma which means in small amounts, between two different times, there must be an evaporation or in the other name vibration decrease since free space has resistance, and it is recovered since there will be friction or collision by different magnitudes as non-flexible; so repeat and thus frequency required to keep matter standing. A work is constantly done even in each small time. Matter is sum of many frames.

As you can see over the above stated derivative components, $\mathrm{dx}, \mathrm{dy}, \mathrm{dz}$ are the smallest 3D derivative components of an unknown function whatever the function is; so as matter basically works over the same principle, namely if that components were for speed, distance, force or time by changing functions since the rule is going to be the same, for example if you knew $t$ as the same for any component during measuring distance over $\mathrm{x}=\mathrm{vt}$, then $\mathrm{v}$ would be different. If you knew v, then $t$ would be different. As also there is time differences, a motion is only able to emerge over arcs since there is no middle point for any force applied; so additionally if you know $t, v$ becomes uncertain because of emerging irrationality; so in the exact opposite way of the measurement over the wave function in accordance with Heisenberg, not only at the same time, even if you measure any physical value at different times even for different phenomenon, there is going to be no intersection point. You may get closer but equation. As a result, 3D position or for example 3D mass cannot be detected certainly if you know for example time. Even you cannot detect the other component if you know one of them. Namely, the uncertainty is not limited by momentum and destination. It is also valid for example between mass and destination or time and distance. As a result for like-this relations, you must relate them by both inequality and uncertainty like Heisenberg did. Also you can write your own with coordinate system.

This is like the rule of circle. If you take the circumference as integer, then you make the radius uncertain as irrational. If you take the radius as integer, then the circumference becomes uncertain because of the relation between area and length of the circle.

Hence entropy also increases as long as there is motion. If you assume that there are infinite universes then the total energy of the universe which is done to create it against space resistance must be provided by another universe or energy source. It means between two energy, there is an energy transfer. While our universe is emerging, the source loses energy, and when our universe gets lost, the energy stored again by the source; but as you can see this is not different then the energy transfer in our universe during creation or after creation. Namely, if there is a transfer, there is going to be vibration decrease and extra energy need. It means, the source is consumed by $\mathrm{mc}^{2}$ each second because of both on-space entropy which emerge during constantly creation repeat of universe and the entropy which is equal to existence of matter since the work-done is done against extinguishedness of matter. It means our universe consuming many universes, as the other universes consume many other that also source universes need energy from other one, then where is the extra energy provided from. Our universe also must be source of another one.

If you think there are limited universes that the number can be like $10^{10^{10000}}$, as infinity can never work since anything is element of it namely there is no energy change in infinite energy, these universes would spend $\mathrm{mc}^{2}$ even in infinite sum. Namely energy spent by these universes for 1 second is equal to infinite period of their life time spending. As a result there cannot exist infinite universes at the same time with infinite transfer. Infinite transfer amount means emergence from nothingness constantly each second since requires infinite energy decrease to 0 and then increase from 0 . This is non-sense. Already it cannot be over time. It happens untimely manner and first time creation is not possible.

Already as you can see, there is no constant speed due to the components. It also means there is acceleration or deceleration. Acceleration is only possible from zero point that is not possible; but deceleration is possible from infinite, absolute energy, and emerging things are its virtual parts with frequency. There is an imaginary time point that I proved it mathematically [1]. There are imaginary time, motion and energy.

We should develop a new probability function over conservation of area and thus of energy. Even we should include appearance from imaginary time which I discovered that you can check it over the rule of the triangle which proves impossibility of perpendicularity, and seems also Riemann surfaces are dominant in universe. Complex analysis is required.

The classic probability function which is based on Gaussian normal distribution allows even infinite universes or for example taking place at infinite distance. Actually these must be between some intervals. Namely if you have a probability detector, and if you take place at Mars, for an electron which is placed at the world somewhere, the probability will increase as long as you get closer to the world. If you take place at Jupiter, just the amount changes but again converges as long as you move towards the world; but you cannot take place at somewhere beyond the universe itself since the area can only hold limited energy. The probability is limited. There are some paradox. Instead of it, ratio of each probability increases and cannot be certain or 1 none of them alone; but can 
be 0 and this is paradox of physical laws. In a limited interval there are infinite probabilities, times, and this is dependent of irreversibility of entropy and thus single way of time. In the same manner, the place which the electron takes place cannot be 0 . As a result, a function to use for probability calculations, it cannot be integrated to infinity and from negative infinite.

I am going to work about these. If you find this short work helpful, also you should in the name of perfection of our abilities and information.

\section{References}

1. Kavak M. 2018, Complementary Inferences on Theoretical Physics and Mathematics, OSF Preprints, Available online: https://osf.io/tw52w/

2. Kavak M. 2018, Gravitational Catastrophe and Dark Matter, OSF Preprints, Available online: https://osf.io/wphxn/

3. Kavak M. 2018, Is Time Misconception of the Transformations Possible?, OSF Preprints, Available online: https://osf.io/edsyj/ 